\title{
HÉRACLÈS ET LES SCYTHES DANS LA MÉMOIRE DES GRECS DE LA MER NOIRE. QUELQUES RÉFLEXIONS SUR HÉRODOTE, IV, 8-10
}

\author{
Herakles and the Scythians in the Greek memories on the \\ Black Sea region. Thoughts about Herodotus, IV, 8-10
}

\section{Lucio Maria Valletta ${ }^{2}$}

\begin{abstract}
RÉSUMÉ
Parmi les différents récits au sujet des origines du peuple des Scythes (Hdt. IV, 5-15), Hérodote nous présente aussi celui retenu par les Grecs de la mer Noire (ch. 8-10). Ce morceau tout entier du texte d'Hérodote s'avère plutôt intéressant, pas tant dans le but d'établir une espèce de descendance d'un archétype unique de mythes et/ou de personnages qui sont devenus ensuite l'expression de patrimoines identitaires de peuples différents, que pour réfléchir sur l'existence d'éléments culturels - détenus par des peuples ayant vécu et se déplaçant dans les régions qui entouraient le bassin de la mer Méditerranée, y compris la mer Noire - qui étaient partagés par ces mêmes peuples que les caractéristiques environnementales avaient vraisemblablement mis en contact. Si, en effet, le récit de la naissance du peuple scythe retenu par les Scythes eux-mêmes offre des éléments (e. g. le nom du premier roi des Scythes, Colaxaïs) qui permettent, par exemple, d'établir quelque lien entre la civilisation des Scythes et celle des Spartiates de l'époque archaïque (le cheval " colaxéen » mentionné dans Alcm. fr. 3, 59 Calame), le récit retenu par les Grecs de la mer Noire (ch. 8-10), qui a comme protagoniste Héraclès, semble quant à lui mettre en évidence des éléments (e. g. l'arc, l'union avec une créature surhumaine)
\end{abstract}

1 J'aimerais remercier vivement François de Polignac de m'avoir invité à réfléchir encore sur certains passages de mon texte et à les préciser davantage. Je reste en tout cas le seul responsable de son contenu. Je remercie aussi les éditeurs du volume d'avoir voulu accueillir cette étude dans leur recueil et de m'avoir fait des suggestions utiles en vue de sa publication.

2 Post Doc researcher: École Pratique des Hautes Études, Section des Sciences Religieuses. E-mail: luciovalletta@virgilio.it 
partagés avec certains poèmes des civilisations du Proche-Orient (e. g. le poème d'Aqht, Ugarit ; le poème de Gilgamesh), revenant d'ailleurs aussi dans l'Odyssée.

Mots-clés : mémoire, épopées archaïques, réseau de relations, éléments culturels partagés

\begin{abstract}
Among the various accounts concerning the origins of the people of the Scythians (Hdt. IV, 5-15), Herodotus also presents to us that retained by the Greeks of the Black Sea (ch. 8-10). This entire piece of Herodotus' text is rather interesting not so much in order to establish a kind of descent from a single archetype of myths and / or characters which then became the expression of identity heritages of different peoples, but rather to reflect on the existence of cultural elements - held by peoples that lived and moved in the regions which surrounded the basin of the Mediterranean Sea, including the Black Sea - which were shared by these same peoples that the environmental characteristics had clearly put in reciprocal contact. If, in fact, the account of the birth of the Scythian people retained by the Scythians themselves offers us elements (eg. the name of the first king of the Scythians, Kolaxaïs) which, for example, allow us to establish some link between the civilization of the Scythians and that of the Spartans of the archaic period (the "Kolaxian" horse mentioned in Alcm. fr. 3, 59 Calame), the account retained by the Greeks of the Black Sea (ch. 8-10), and which has Heracles as protagonist, seems on the other hand to offer us elements (eg. the arc, the union with a superhuman creature) shared with poems from civilizations of the Near East (eg. the poem of Aqht, the poem of Gilgamesh), and who also come back in the Odyssey.
\end{abstract}

Keywords: memory, archaic epic, people networks, shared cultural items

\title{
1. Introduction
}

Sous quelle lumière pourrait-on observer le personnage d'Héraclès, à travers les phases qui caractérisent sa vie ${ }^{3}$, au fil de ses entreprises qui le conduisent dans des régions du monde grec et ensuite méditerranéen à une

3 Très bien mises en ordre pour nous dans des ouvrages tardifs tels que la Bibliothèque du ps. Apollodore (II, 4, 8-7, 8 [61-166]) et la Bibliothèque Historique de Diodore de Sicile (IV, 8-39), ainsi que dans une série de poèmes plus anciens qui lui étaient dédiés et qui, malheureusement, ont étés perdus (voir ci-dessous). 
échelle de plus en plus large ? Est-ce qu'il faut remarquer, par exemple, que c'est bien à l'occasion de certaines de ses entreprises que le roi du pays donne sa fille en épouse à Héraclès - lui permettant d'avoir une descendance et ainsi de jeter les bases d'une nouvelle dynastie locale qui remonte à lui ? On relève que :

- c'est le cas de Mégara - la fille du roi de Thèbes Créon (Diod. IV, 10 ; ps. Apollod. II, 4, $11[70])$;

- c'est le cas d'Iole - la fille d'Eurytos, roi d'Oichalia (Diod. IV, 31, $1-2 ; 37,5$; ps. Apollod. II, 6, 1 [127] et 7, 7 [156]) ;

- c'est le cas de Déjanire - la fille d'Oineus roi de Calydon (Diod. IV, 34, 1 ; 36, 1 ; ps. Apollod. I, 8, 1 [64] ; II, 7, 8 [165]).

Ces cas sont seulement quelques-uns des plus célèbres parmi bien d'autres qui représentent, pour ainsi dire, la trace permanente du passage d'Héraclès dans une région donnée, à côté des effets que son exploit ( civilisateur » ?) dans cette région implique pour l'avenir. On peut aussi rappeler le cas des Héraclides qui régnaient en Lydie (Hdt. I, 7), auxquels Héraclès donne origine dans le contexte de son esclavage chez Omphale (TALAMO, 1979). Afin de comprendre toutes les implications d'un personnage si fécond (au prisme de la génération de ses progénitures aussi bien que de mythes qui faisaient référence à lui), je crois que le cas du récit de l'origine du peuple des Scythes doit, lui aussi, être tout d'abord mis en série avec les autres unions d'Héraclès avec des personnages féminins. C'est seulement ensuite - tout en tenant compte de cela, et de l'ample échelle d'action d'Héraclès parmi les héros grecs - qu'on pourrait évaluer les éléments particuliers de ce récit qui permettent aussi d'établir une comparaison entre le personnage d'Héraclès au milieu de la civilisation grecque et d'autres 
héros au milieu des civilisations méditerranéens et proche-orientales avec lesquelles les Grecs avaient eu des contacts au fil de leur histoire ${ }^{4}$.

Dans une espèce de réflexion circulaire, on commencera donc par une analyse des éléments constitutifs du récit qu'Hérodote nous fournit au sujet du rôle d'Héraclès dans le processus qui aboutit à la naissance des Scythes - tout en ramenant cet épisode au contexte plus large de la vie d'Héraclès et des moments qui caractérisent les différentes étapes (notamment son entraînement durant la jeunesse, et l'arc de sa vie d'homme mûr). Ensuite - loin d'aborder toute hypothèse qui implique une origine orientale du personnage d'Héraclès lui-même -, on élargira le regard à certains éléments et épisodes analogues qui concernent certains des héros protagonistes des légendes façonnées par les civilisations du Proche-Orient. On reviendra, en conclusion, au regard des Grecs sur ces mêmes éléments de culture que le cas du mythe de la naissance des Scythes nous présente, pour proposer une image d'Héraclès en tant que héros à la nature « corrompue ».

4 Je fournis ici une carte de l'ancien territoire des Scythes, dans le contexte géographique du bassin de la mer Noire (carte réalisée par moi-même en indiquant les noms des mers, des régions et des peuples, à partir d'une image de la zone géographique du bassin de la mer Noire prise par Google Earth Pro) :

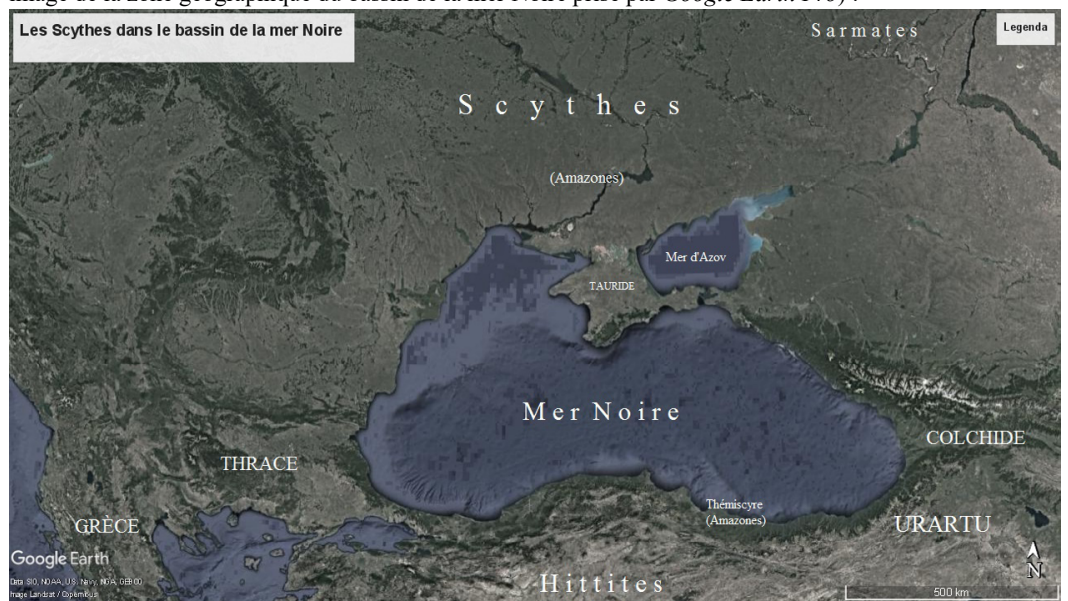




\section{Le mythe de la naissance des Scythes chez les Grecs dans le contexte de la légende d'Héraclès}

Dans la version du mythe retenu par les Grecs au sujet de l'origine du peuple des Scythes transmise par Hérodote (Hdt. IV, 8-10), il est possible d'isoler certains éléments qui nous seront utiles en vue d'une comparaison (aussi sous l'aspect de la chronologie des récits qui nous en transmettent les mythes) entre ce héros grec et d'autres personnages proches du monde grec tout en lui restant étrangers. En particulier :

a) les circonstances dans lesquelles Héraclès serait arrivé sur la terre ensuite peuplée par les Scythes (lorsqu'il poussait devant lui les vaches de Géryon ; 8,1);

b) l'hiver et le froid - et le sommeil qui prend Héraclès dans cette région $(8,3)$;

c) la recherche des cavales qu'Héraclès avait attachées à son char $(8,3-9,1)$;

d) le personnage de la créature à la double nature de jeune fille et de serpent, qui régnait sur le pays - à laquelle Héraclès s'unit pour qu'elle lui rende les cavales qu'elle avait retenues $(9,1-2)$;

e) la conception de trois enfants à la suite de cette union - enfants d'Héraclès et de la souveraine monstrueuse de la région - parmi lesquels on aurait dû choisir celui qui, premier roi du pays, aurait aussi assuré par son propre lignage un gouvernement durable du territoire $(9,3-5 ; 10,3)$;

f) les éléments de l'arc et de la ceinture (cette dernière ayant une phiale d'or à son extrémité, en correspondance de la fermeture) outils pour discerner celui qui aurait été apte à obtenir cet honneur $(9,5$; le frère cadet Scythès ; 10, 2). 


\section{A. Les circonstances}

L'entreprise de lui amener les vaches de Géryon d'Ibérie (ps. Apollod. II, 5, 10 [106] ; Diod. IV, 17-24) est la dixième épreuve qu'Eurysthée impose à Héraclès pour que ce dernier puisse obtenir l'immortalité (ps. Apollod. II, 4, 12 [73] ; Diod. IV, 10, 7). On pourrait dire - de manière très synthétique - que la période qu'Héraclès passe à accomplir les travaux qui lui sont imposés par Eurysthée marque précisément le passage du personnage de sa dimension régionale originaire (l'Argolide, la Béotie) et de sa condition de jeune guerrier au statut d'homme mûr (à la suite, également, du mariage avec Mégara; ps. Apollod., II 4, 11 [70]; Diod. IV, 10, 6) et à une échelle spatiale de plus en plus large au fur et à mesure qu'Héraclès surmonte les épreuves. C'est bien à ce moment-là - d'après la Bibliothèque (ps. Apollod. II, 4, 12 [73]), à la différence de Diodore (Diod. IV, 10,1) - qu'Héraclès est appelé pour la première fois par la Pythie avec ce nom ${ }^{5}$ qui l'identifiera partout dans la mémoire des Grecs, tout en oubliant le nom d'Alcide 6 .

À partir des régions plus proches de ses lieux d'origine (Némée en Argolide, où Héraclès tue le lion dont il porte ensuite la célèbre peau (SCHNAPP-GOURBEILLON, 1998, p. 98-112) ; Lerne, en Argolide également) et incluses dans le Péloponnèse ${ }^{7}$ (Cérynie en Achaïe ; le mont Erymanthe en Arcadie ; l'Elis, royaume d'Augias ; le lac Stymphale), les épreuves qu'Héraclès doit surmonter et accomplir sur ordre d'Eurysthée l'amènent jusqu'aux régions plus éloignées du bassin de la mer Méditerranée (l'île de Crète ; la Thrace ; la côte méridionale de la mer Noire ; les régions ibériques près de l'Océan) - tandis qu'on pourrait distinguer au moins

$5 \mathrm{Au}$ sujet du lien que ce nom établit avec Héra, voir e. g. PIRENNE-DELFORGE ; PIRONTI, 2016, p. 264-77.

6 Voir e. g. STAFFORD, 2012, p. 8-9. Il ne faudrait pas négliger la valeur euristique de la correspondance entre ce changement de nom et ce moment particulier de la vie d'Héraclès, jusqu'à aboutir à la fonction représentative et, pour autant, symbolique du langage (identifiant aussi la relation entre sujet et objet), à l'origine de l'attribution des noms, mise en lumière au moins à partir d'Ernst Cassirer (1923). Sans, donc, aborder ici la question en profondeur, on relève que, dans l'Antiquité, le changement du nom personnel se vérifiait pour plusieurs raisons, telles que l'acquisition de prestige par un certain individu au milieu de sa propre communauté (voir, très récemment, PARKER [éd], 2019). Au sujet des changements de nom à la suite d'un changement du statut de l'individu au milieu de son groupe humain dans les sociétés tribales du Nord-Ouest américain, voir MAUSS, 1938 (1950), p. 341-44. Pour ce qui concerne le cas d'Héraclès, voir notamment BRILLANTE, 1992, p. 212-13.

7 À ce sujet, voir LÉVÊQUE ; VERBANCK-PIÉRARD, 1992, p. 45, qui parlent d'une vaste opération de katharsis du Péloponnèse. 
deux parcours qui se déroulent et s'entrelacent entre eux de manière réciproquement dépendante dans la construction du personnage d'Héraclès en tant que héros des Grecs :

- l'arc de la biographie personnelle de ce personnage, qui le conduit - à la fin de sa vie - à obtenir l'immortalité ;

- la découverte progressive de la part des Grecs, par le biais des événements de cette biographie personnelle, du " tout petit monde » (MALKIN, 2011) ${ }^{8}$ représenté par le bassin de la mer Méditerranée, y compris la mer Noire.

La fonction d'Héraclès (vainqueur de créatures monstrueuses ou de peuples à la vie marquée par une forme de démesure) et le processus que son action déclenche là où auparavant une forme de désordre subsistait peuvent ainsi être observés - au niveau de la représentation fabriquée par les Grecs dans leur mythes - comme le regard que les Grecs eux-mêmes portaient sur des régions et des peuples qui auparavant leur étaient inconnus (une autre façon, si on veut, de parler au sujet d'Héraclès de « miti di precedenza $\left.»^{9}\right)$. Il faut pour cela considérer que la légende d'Héraclès est déjà façonnée à la fin de l'époque archaïque - et le héros est bien présent aussi dans les poèmes homériques. Hérodote lui-même (Hdt. II, 44) était convaincu de la haute ancienneté d'Héraclès (notamment en tant que dieu égyptien et phénicien) - et, d'après certaines études ${ }^{10}$, le héros grec devait être au moins mycénien à l'origine ${ }^{11}$.

8 On fait allusion ici au titre de la traduction française (2018).

9 Au sujet de la notion de « miti di precedenza », voir notamment GIANGIULIO, 1983 et, par rapport au récit de la naissance des Scythes dans le passage d'Hérodote, voir le commentaire d'Aldo Corcella (1993, p. 234). Voir aussi LÉVÊQUE; VERBANCK-PIÉRARD, 1992, p. 45.

10 E. g. NILSSON, 1932, p. 219. De son côté, Walter Burkert (1998, p. 12) doit admettre que les origines d'Héraclès restent introuvables et qu'il n'existe pas de témoignage mycénien direct, ni écrit ni figuré, même si les reconstructions ne font pas défaut. D'ailleurs, Paul Wathelet (1998, p. 52 et $60 \mathrm{n}$. 1), en faisant confiance à certaines recherches de C. J. RUIJGH et de J. DRIESSEN, souligne le cas - dans le texte d'Homère - de

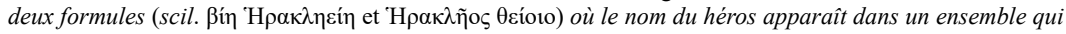
remonte au moins au temps des tablettes en linéaire $B$ (p. 52) et le fait qu'il est possible sans plus que le nom d'Héraklès soit attesté sur une tablette mycénienne de Cnossos KN Xd 305 lra-ke-re-wel (p 60 n. 1).

11 Voir aussi LÉVÊQUE ; VERBANCK-PIÉRARD, 1992, p. 44-45, qui observent que (p. 45) ce n'est sans doute pas avancer beaucoup que de reconnaître en Héraclès un héros mycénien (avec référence, évidemment, au modèle de héros). Malheureusement, nous ne disposons pas (à l'exception de quelques fragments) de plusieurs ouvrages anciens que les poètes lui avaient consacrés - tels que les Herakleia de Pisandre de Rhodes (fin du VII ${ }^{\mathrm{e}}$ siècle av. notre ère), ou les Herakleia de Panyassis d'Halicarnasse (moitié du Ve siècle av. notre ère) - desquels, néanmoins, il faut tenir compte du fait que plusieurs éléments aient conflué dans des compositions comme la Néméenne I de Pindare ou l'Idylle XXIV de Théocrite. 
Ainsi, l'entreprise d'amener les vaches de Géryon - localisée dans l'Occident extrême, au-delà des piliers d'Héraclès, ce qui lui ouvrira aussi « les portes du soir » (JOURDAIN-ANNEQUIN, 1989, p. 517-67 et MARIOTTA, 2012, p. 71), c'est-à-dire l'au-delà duquel il va ramener Cerbère (Diod. IV, 25) - implique-t-elle néanmoins, pour être accomplie, qu'Héraclès conduise les bêtes à travers un long chemin qui passe aussi par certaines régions les plus extrêmes de l'Orient méditerranéen, et donc par le territoire des Scythes. Cela est d'autant plus intéressant si on tient compte que nous retrouvons le mythe de Géryon présenté de manière synthétique déjà chez Hésiode (Th. 287-94 et 979-83) - il devait donc être déjà connu au moins par les communautés des régions (y compris la Béotie, de laquelle Hésiode était originaire) qui étaient aussi les lieux auxquels Héraclès était plus étroitement lié.

\section{B. L'hiver, le froid et le sommeil}

Dans le cadre de cette connotation sauvage de la région de la Scythie, dans laquelle Héraclès arrive, nous relevons également les détails de l'hiver et du froid qui surprennent le héros, ainsi que le sommeil qui s'ensuit, auquel Héraclès s'abandonne. Dans la légende d'Héraclès, le sommeil arrive parfois à la suite d'un moment d'angoisse physique ou mentale (PAPADOPOULOU, 2005, p. 68, qui mentionne aussi le cas d'Oreste, Eur. Or. 166-86) - comme dans le cas de la folie qui pousse Héraclès à tuer ses fils (et parfois aussi sa femme Mégara ; Eur. $H F, 1004-06)$ - lui apportant un soulagement, temporaire ou définitif, par rapport aux efforts qui l'ont précédé12. Mais le Sommeil - frère de la Mort chez Homère (Il. XIV 231) aussi bien que chez Hésiode (Th. 212-13) - se révèle aussi, dans la pensée des Grecs, comme un agent trompant et affaiblissant la volonté humaine ${ }^{13}$ - même dans le cas d'un dieu souverain tel que Zeus (Hom. Il. XIV, 157-65 ; 231-62 ; 346-53). D'ailleurs - comme dans le cas célèbre de la Dios apatē dans l'Iliade que

12 C'est aussi le cas, très fréquemment, du sommeil dans l'Iliade.

13 Pour ce qui concerne Héraclès, voir e. g. PAPADOPOULOU, 2005, p. 121 et STAFFORD, 2012, p. 118-20. 
nous venons de mentionner, où l'action du Sommeil est complémentaire à celle d'Aphrodite (PIRENNE-DELFORGE ; PIRONTI, 2016, p. 42-46) -, on note que l'action affaiblissante du sommeil est liée de quelque manière (en tant que la prépare) à l'union sexuelle entre Héraclès et la créature à la double nature que nous allons analyser d'ici peu.

\section{Les cavales}

Avant d'en arriver là, c'est aux cavales et à leur recherche qu'il faut consacrer quelques observations. Même dans le cas où il s'agirait simplement d'un élément de la culture scythique qui entre dans le mythe fabriqué par les Grecs de la mer Noire ${ }^{14}$, nous apprenons que la VIII épreuve qu'Eurysthée impose à Héraclès (Diod. IV, 15, 3-4; ps. Apollod. II, 5, 8 [96] ; voir aussi Eur. Alc. 482-504) est de lui amener de Trace les cavales de Diomède - le fils d'Arès, roi du peuple des Bistons - et qu'Héraclès luimême avait reçu de Poséidon des chevaux en cadeau (Diod. IV, 14, 3) qu'il aurait pu atteler à son char.

Par conséquent, il faut retenir cet élément aussi-de même que l'arc et la ceinture (voir ci-dessous) - comme l'un des attributs qui définissent la fonction d'Héraclès en tant que héros des Grecs - une fonction que nous sommes en train de reconstruire au fur et à mesure que nous avançons dans notre analyse et qui concerne l'une des plus anciennes formes saisissables de la royauté en Grèce et, pourrait-on dire, chez plusieurs peuples de la Méditerranée (y compris les Scythes), celle du « chef guerrier $\rangle^{15}$, qui est encore celle des poèmes homériques ${ }^{16}$. Héraclès, d'un côté, rend donc dociles les cavales de Diomède (féroces auparavant jusqu'à dévorer les

14 Voir CORCELLA, 1993, p. 235, d'après ALY, 1969, p. 120-22. C'est bien dans ces mêmes régions de la mer Noire (y compris la Scythie, notamment au $\mathrm{VI}^{\mathrm{e}}$ siècle av. notre ère) que vivaient les Amazones, contre lesquelles Héraclès entreprit une expédition pour rapporter à Eurysthée la ceinture de leur reine Hyppolite (Diod. IV, 16 ; ps. Apollod. II, 5, 8 [97]). Pour le cas des Amazones, voir en général, très récemment, MAYOR, 2014.

15 À ce sujet - hors de toute schématisation trifonctionnelle, pour ce qui concerne mon interprétation - voir BADER, 1992, p. 21-22 et 1998.

16 Voir aussi LÉVÊQUE ; VERBANCK-PIÉRARD, 1992, p. 45. 
humains) en les rassasiant avec la chair de celui qui leur avait appris à violer les usages (Diod. IV, 15, 3) et, par ailleurs, maîtrise celles qu'il avait attachées à son char $^{17}$.

\section{L'être à la double nature et l'union avec Héraclès}

Le moment qui suit - celui de l'union d'Héraclès avec cet être à la double nature, qui avait retenu les cavales du héros - est ainsi configuré comme la rencontre entre ce modèle culturel de la royauté plus ancienne représenté par Héraclès (qui, à la suite de toutes les entreprises accomplies, a désormais acquis son statut de héros mûr, qui lui était reconnu aussi par les dieux ; voir notamment Diod. IV, 14, 3) et la dimension que l'on dirait étrangère à toute condition favorable à la vie humaine ${ }^{18}$, représentée par cet être féminin en termes d'absence de toute trace identifiable de civilisation aussi bien que de condition demi-animale. Ainsi, cette union nous permet de saisir aussi pour Héraclès - à côté de ce modèle de royauté très archaïque le deuxième modèle auquel nous avons fait allusion, c'est-à-dire celui que P. Lévêque (LÉVÊQUE ; VERBANCK-PIÉRARD, 1992, p. 45) définit de héros cosmique qui purge le monde et repousse les limites de l'oikoumène par ses voyages de découverte.

Mais un élément comme l'union avec cette créature féminine révèle que c'est en tant que modèle de héros du premier type (celui du chef archaïque porteur d'un système d'éléments culturels partagés) qu'Héraclès peut remplir aussi la fonction de ce deuxième type de héros. Ces deux aspects ne sont donc pas séparables. C'est aussi le cas d'autres personnages appartenant à la légende héroïque des Grecs - tels que Jason ou Ulysse, ou encore Phrixos - durant leurs voyages (qu'il s'agisse d'échapper à la mort donnée par la main de son propre père [Phrixos], avec ce que l'on peut lire comme un symbole de royauté, c'est-à-dire le bélier à la toison d'or ; ou de récupérer

17 Voir notamment BADER, 1998 et, en général, pour ce qui concerne la domestication des animaux, BURKERT, 1992, p. 116-17.

18 Voir BURKERT, 1992, p. 113-16, qui parle aussi d'Héraclès en termes d'« organisateur du paysage ». 
la toison d'or pour obtenir à nouveau cette dignité royale [Jason]; ou encore de revenir à son propre royaume [Ulysse]). Le cas de l'union entre Jason et Médée et celui du séjour d'Ulysse sur l'île de la nymphe Calypso (Hom. Od. V et VII, 245-60) ou sur celle de Circé (Hom. Od. X) sont célèbres, certes, mais c'est aussi le cas du mariage de Phrixos avec Chalciopè, fille du roi de Colchide, Aiétès (ps. Apollod. I, 9, 1 [83]).

Par ailleurs, à propos du personnage féminin impliqué dans ce mythe « scythique » d'Héraclès, on a fait allusion à une forme de la " grande déesse » de la fertilité ou de la nature, dont on a plusieurs représentations chez les Scythes (CORCELLA, 1993, p. 235), et on a aussi mis en relation sa double nature avec celle du personnage d'Échidna (Hes. Th. 295-305), aussi bien qu'avec plusieurs personnages féminins des légendes orientales que nous allons analyser plus loin. D'ailleurs - pour ce qui concerne le monde grec, au lieu de penser à une figure « archétypale » de « déesse de la nature $\rangle^{19}$-, on pourrait rappeler plutôt certaines manifestations d'Artémis (comme la "sauvagerie » de la Tauropolos (ELLINGER, 2009, p. 207-

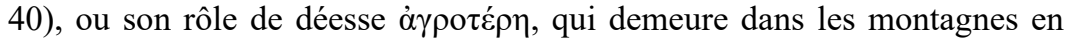
se réjouissant de la chasse (H. Hom. XXVII [in Dian.], 1-10), par rapport à l'épisode héracléen de la biche de Cérynie (ELLINGER, 2009, p. 3956) ; ps. Apollod. II, 5, 3 [81-82]), ou encore certaines d'Aphrodite (comme l'Ourania - par rapport e. g. à sa fonction « primordiale » de puissance de la mixis et des humeurs vitales (PIRONTI, 2005 et 2007, p. 51-67, 67-100 et 153-208); si on tient aussi compte e. g. de Hdt. IV, 5920). Et, par rapport à tout cela, il est aussi intéressant de remarquer - au contraire - le rôle central qu'une déesse telle qu'Athéna jouait dans la légende d'Ulysse autant que d'Héraclès. Encore, au sujet des deux régions de la Colchide et de la Tauride, dans le contexte septentrional et oriental de la mer Noire de même que les Scythes (qui avaient établi des alliances avec les Colques ; voir notamment Diod. IV, 47, 4) - il est intéressant d'observer (Diod. IV, 45-47) que les deux fils d'Hélios, rois de ces deux régions (Aiétès en Colchide ; Persée en Tauride) avaient, eux aussi, pour trait commun la cruauté, et qu'Aiétès, en plus de Médée, avait aussi comme fille précisément Circé $^{21}$. Si on considère

19 Une idée archétypale qui ne reste, en tant que telle, rien de plus qu'une abstraction.

20 Voir, à ce propos, aussi CORCELLA, 1993, p. 281-82 et PIRENNE-DELFORGE, 2005, p. 274-79.

21 On relève d'ailleurs que, d'après une tradition alternative (e. g. Hes. Th. 956-57), Circé était la sœur d'Aiétès. D'après cette même tradition - suivie aussi par la Bibliothèque du ps. Apollodore -, on a vu que la deuxième fille d'Aiétès était Chalciopè. 
ces façons différentes de représenter l'altérité de ces régions - notamment en relation à la façon selon laquelle est construite la venue des héros grecs (Phrixos ; Jason ; Ulysse ; mais aussi Oreste ${ }^{22}$ ) dans ces régions, d'après les récits qui les concernent -, je crois que cela éclaire mieux aussi la légende d'Héraclès, dans la mesure où s'interroger sur chacun de ces cas parallèles nous aide à comprendre l'articulation du personnage d'Héraclès en tant que héros pour les Grecs. C'est ainsi, en effet, que nous arrivons au cœur de la question, par rapport à notre perspective d'enquête.

Cela veut dire que, si nous tenons compte d'une conception plurielle de ce qu'était en effet un dieu ou un héros ${ }^{23}$ - comme Louis Gernet (GERNET ; BOULANGER, 1932 (1970), p. 222) lorsqu'il affirmait que positivement, un dieu est un système de notions, ou Jean-Pierre Vernant (1960 (1965), p. 362) qui observait que les dieux helléniques sont des Puissances, non des personnes et que chaque puissance n'a réellement pas $d$ ' "existence pour soi », mais exclusivement par le réseau de relations qui l'unit au système divin dans son ensemble. Et dans ce réseau elle n'apparaît pas nécessairement comme un sujet singulier, mais aussi bien comme un pluriel -, on s'aperçoit alors que chaque tentative d'identification se révèle elle-même l'outil d'une méthode difficilement praticable (aussi du fait du risque - qui se cache derrière toute identification - $d$ 'en tirer une quelconque catégorie « archétypale »).

Il est toujours utile, je crois, de se souvenir de ce que Vernant a voulu expliquer de sa méthode dans l'un de ses derniers entretiens en 2003 (LE GOFF ; VERNANT, 2014, p. 32-34), lorsqu'il précisait (par rapport à l'approche structuraliste de Lévi-Strauss) qu'il existe un ordre du récit et qu'une architecture se dégage, mais que d'ailleurs je ne suis pas sûr qu'on puisse passer sans prendre beaucoup de précautions des mythes grecs à des mythes africains, amérindiens, en concluant ainsi (faisant allusion à la méthode de son maître Ignace Meyerson) que la psychologie est historique. Dans le cas des mythes dont nous nous occupons ici, il est vrai que les peuples qui les ont produits avaient plusieurs occasions de contacts et échanges mais - au lieu de penser à une identification tout court entre tous ces personnages (les dieux aussi bien que les héros) - il faudrait penser à une analogie plutôt

22 Notamment par rapport au rôle qu'il satisfait, e. g., dans l'Iphigénie en Tauride d'Euripide. Voir aussi, pour ce qui concerne la présence de la statue cultuelle d'Artémis Orthia à Sparte, Paus. III, 16, 7.

$23 \mathrm{Ce}$ qui devient évident par leurs différentes appellations (épithètes/épiclèses). 
sous l'aspect des fonctions ${ }^{24} /$ notions $^{25}$ que chacun satisfaisait au milieu d'un " ordre du récit » qui, pour autant, était également semblable pour chaque mythe. C'est bien cela qui nous intéresse, c'est-à-dire de comparer les manières différentes selon lesquelles chaque civilisation faisait face à des exigences analogues.

\section{E. La conception des trois enfants}

Ce qui compte le plus ici - dans le cas des mythes grecs aussi bien que proche-orientaux - est d'examiner le sens et la valeur de ces unions (qui parfois se réalisent sous la forme d'un véritable mariage légitime), dans des contextes où la fonction d'élargissement des horizons de sa propre civilisation se lie aussi à la nécessité - comme M. Finkelberg aussi (2005, p. 65-89 et p. 90-108, qui prend aussi en compte l'étude de Louis Gernet au sujet des mariages de tyrans, 1954 [1968]), assez récemment, l'a bien mis en évidence - d'assurer la survivance de son propre lignage au moyen d'alliances à établir avec des dynasties enracinées ailleurs ${ }^{26}$. Dans ces processus c'est souvent le personnage féminin qui garantit la continuité par rapport au territoire alors que le personnage masculin, lui, " transfère », pour ainsi dire, sa propre dignité dynastique à la femme à laquelle il s'unit (FINKELBERG, 2005, p. 69-71).

24 Ici, je reprends le mot-clé du titre de l'ouvrage d'I. MEYERSON, Les fonctions psychologiques et les ouvres, Paris : Vrin, 1948

25 Ici, je reprends le mot-clé utilisé par L. GERNET dans la définition de ce qui est un dieu que je viens de citer. 26 En particulier, par rapport à la perspective spatiale impliquée par ces mythes de voyage et d'aventure, moi aussi, je tiens compte de trois éléments différents au moins qui - en relation réciproque - agissent en déterminant la formation de ces réseaux au fil des générations :

- les conditions d'un contrat de mariage légitime entre les nobles ;

- la possibilité qui s'ensuit de prolonger de quelque manière le pouvoir de sa propre lignée :

o soit qu'il s'agisse de garder son propre pouvoir dans le cas d'un garçon qui n'a pas accès au pouvoir de son père ;

o soit qu'il s'agisse d'agrandir le réseau de relations ou de prolonger le pouvoir de la lignée de son père ;

- le phénomène - implicite à la nécessité que ce pouvoir s'exerce dans un territoire défini, par rapport aux deux éléments qui précèdent - du déplacement du pouvoir d'un certain lignage d'un lieu à l'autre, dans des formes qui impliquent aussi celles (connues aussi par Héraclès) de l'exil ou de la colonisation. 
Et c'est bien ce qui se passe ici lorsqu'Héraclès engendre sa descendance avec la créature à la double nature, en lui confiant les symboles de son pouvoir afin qu'elle puisse les transmettre (avec le pouvoir qu'ils symbolisent) à celui d'entre leurs futurs enfants qui - devenu adulte - sera capable de réussir les épreuves données : tendre l'arc et ceindre la ceinture de son père ${ }^{27}$ de manière qu'il puisse régner sur la terre de sa mère. La valeur symbolique de l'arc est évidente du fait de l'épreuve célèbre à laquelle, dans l'Odyssée (Hom. Od. XIX, 570-81 ; XXI, 1-12 et 68-79), Pénélope soumet les prétendants au pouvoir de son époux - qui serait ainsi transféré à la maison du vainqueur - et revient aussi dans certains mythes procheorientaux que nous aborderons ultérieurement.

\section{F. Les éléments de l'arc et de la ceinture}

Le détail de cette transmission nous ramène donc aussi au moment de la jeunesse d'Héraclès et à sa paideia, l'entraînement qu'il a reçu. D'après la Bibliothèque (ps. Apollod. II, 4, 9 [63]), nous apprenons que son père Amphitryon (Tirynthe) lui apprend à conduire le char, Autolycos (Ithaque, Thessalie) lui apprend à lutter, Eurytos (Oichalia) à tirer à l'arc, Castor (Sparte) à utiliser les armes, Linos à jouer de la cithare. Le détail révélant que c'est Eurytos qui apprend à Héraclès à tirer à l'arc est d'autant plus remarquable si on considère que l'arc d'Ulysse était aussi un cadeau de son fils Iphitos (Hom. Od. XXI, 13-41; VIII, 223-28) et que, vraisemblablement, c'était un arc scythique. D'ailleurs, il existe une autre tradition (voir e. g. Lyc. Alex. 56) selon laquelle ce serait le berger scythe Teutaros qui aurait appris à Héraclès à tirer à l'arc (BRILLANTE, 1992, notamment les p. 20001 et 206-09), et ce serait bien l'arc que Teutaros lui aurait donné à la suite de cet entraînement que maintenant Héraclès confie à cette créature. Quoi qu'il en soit, ce sont ces mêmes superpositions de personnages et de peuples

27 Comme le relève CORCELLA, 1993, p. 235, c'est aussi le cas de Thésée qui, adulte, reçoit l'épée et les sandales que son père Égée lui avait laissées en héritage (Plut. Thes. 3-ss). Je me pose aussi la question de savoir si la ceinture d'Hyppolite - la reine des Amazones - ait une fonction symbolique semblable, si on tient compte que le territoire des Amazones était inséré dans le même contexte géographique de la mer Noire. 
à l'histoire si différente qui doivent retenir notre attention, en tant qu'elles révèlent précisément cette analogie de fonctions à laquelle nous avons fait allusion et qui - ce qui compte le plus - se traduit aussi dans certaines formes de société partagées qui représentent un véritable « code culturel» (y compris, par exemple, certaines formes du chant ${ }^{28}$ ) pour créer des réseaux de contacts et d'échanges entre civilisations en même temps proches et distinctes ${ }^{29}$.

\section{Héraclès, les Scythes et la royauté spartiate}

Par rapport à la notion de royauté chez les Grecs et les Scythes, par exemple, on connaît la comparaison - à partir du témoignage d'Hérodote que François Hartog (1981 (2001) et 1982) a proposée des funérailles des rois de Sparte (Hdt. VI, 58-60) et des rois Scythes (Hdt. IV, 71-73), tout en soulignant, dans le cas de Sparte, la valeur politique de ces cérémonies (1981 (2001), p. 253-59), aussi bien que de l'héroïsation (Xen. Lak. Pol. 15, 9). À ce propos, on rappelle aussi la description par Hérodote des funérailles des élites de Trace (Hdt. V, 8).

Ces mêmes comparaisons - je me limite à Sparte - ont été aussi le point de départ d'études successives sur la royauté en Grèce. Il me semble que certains chercheurs (e. g. MILLENDER, 2002 e 2009) - en adhérant à la perspective culturelle d'Hérodote - ont plutôt remarqué les traits d'altérité

28 Récemment, sur plusieurs aspects liés aux formes d'adresse aux dieux, voir e. g. METCALF, 2015.

29 Cela veut dire - notamment si on tient compte aussi de ces contacts et échanges au fil de l'âge du Bronze qui, souvent, ne peuvent être observés que grâce à l'analyse des analogies dans la culture matérielle au sens large - qu'il n'est pas question de rechercher des identifications ponctuelles entre les pratiques sociales de ces différentes civilisations (ou entre les différents personnages mythiques qui à leur tour les ont mises en place dans le cadre de leur propre légende). Il s'agit plutôt de saisir la façon suivant laquelle, nécessairement, chaque civilisation a pu développer les traits culturels qui l'identifiaient aussi grâce au partage de pratiques et de savoirs (et aussi d'artéfacts : e. g. l'arc "scythique » gardé par Ulysse dans son palais) avec les civilisations qui lui étaient proches. Cela était en fonction de satisfaire des exigences analogues qui, d'ailleurs, se configuraient entre elles de manière différente au sein de chaque civilisation (ce qui comportait aussi - par rapport à la « fabrication » des mythes - des variations dans ce que Vernant appelait l'« ordre du récit »). Néanmoins, la pratique durable de ces interactions entre civilisations différentes, leur a permis de consolider un système de formes de société semblables (un « code culturel » partagé ; e. g. la chasse ; le banquet ; les pratiques qui impliquaient l'utilisation des chevaux) qui, au fil du temps, s'est révélé être le véritable terrain de communication entre les représentants (e. g. les élites) de ces civilisations. 
(en termes d'archaïsme aussi bien que de barbarie) aussi de l'institution de la royauté à Sparte. D'autres chercheurs (e. g. HINGE, 2003, p. 55-74) au contraire - dans une perspective d'enquête semblable à la nôtre, et tout en tenant compte de la mise en garde de Hartog, c'est-à-dire la possibilité d'expliquer tout cela par le fait que ce sont les Grecs qui décrivent les institutions des Scythes, (y compris les généalogies des Scythes, des Lydiens et des Doriens transmises par Hérodote (I, 7 ; IV, 8-10 ; VI, 52 ; VII, 204 ; VIII, 131), qui ont Héraclès pour ancêtre commun) - ont essayé d'identifier (sous l'aspect de l'archaïsme plutôt que de la barbarie ${ }^{30}$ ) ce « code culturel » partagé auquel nous avons fait allusion.

Par ailleurs, d'après l'analyse conduite par G. Devereux (1965, p. 176-84) des tombeaux des rois scythes, mis au jour près de la localité de Pazyryk dans les contreforts de l'Altaï - où on a aussi retrouvé beaucoup de restes de chevaux ${ }^{31}$, comme Hérodote en témoigne aussi -, Hinge propose une riche série d'hypothèses au sujet des affinités entre les coutumes sociales des Spartiates et celles des Scythes (et pour autant de l'articulation et la fréquence des contacts à l'époque archaïque entre le monde grec et le monde proche-oriental), tout en tenant compte du fait que - dans le mythe de la naissance des Scythes retenu par les Scythes eux-mêmes (Hdt. IV, 5-7) le nom de l'un des fils de Targitaos, Colaxaïs, est le même que celui de l'une des races de chevaux, le cheval colaxéen, qui paraissent dans un des célèbres parthénées d'Alcman (Alcm. fr. 3, 59 Calame). On mentionne aussi les chevaux que Poséidon donne à Pélops - peut-être de la race Enète mentionnée aussi par Alcman (ibidem, v. 51), originaire de la Paphlagonie comme l'était Pélops (voir Diod. IV, 74, 1 et Ap. Rh. II, 358-59) -, ce qui est significatif par rapport à l'institution des Jeux Olympiques, fondés par Pélops et réintroduits par son descendant Héraclès ${ }^{32}$.

On comprend aussi, à la suite d'une pareille configuration de la royauté, la fonction d'Héraclès aux côtés des Dioscures à l'occasion des

30 Un trait que l'on qualifierait de cruel - commun à la royauté grecque archaïque et à celle des monarchies proche-orientales - est le droit du souverain de mutiler ses sujets lorsqu'ils étaient coupables d'une quelconque faute. C'est le cas de l'inscription de Darius à Beshtun $\S \S 32-33$; de Xerxès (Hdt. VII, 39, 3), mais aussi d'Héraclès (Diod. IV, 10, 3) et d'Ulysse (Hom. Od. XX, 474-77).

31 Aussi dans le cas des funérailles de Patrocle, nous apprenons qu'Achille jette sur le bûcher quatre chevaux (Hom. Il. XXIII, 171).

32 Au sujet de l'élevage des chevaux dans la région du « royaume de Priam », d'ailleurs, voir ELLIS-EVANS, 2019, p. 109-53. 
combats des jeunes Spartiates au Platanistas (Paus. III, 14, 8-15, 3 et 20, 2), et on saisit également de quelle manière le personnage d'Héraclès devient le modèle pour représenter la notion de royauté dans un contexte culturel comme celui de l'île de Chypre ${ }^{33}$ - tout en intégrant dans cette représentation de la royauté aussi le motif de son apothéose. Le fait que, dans le contexte local de Sparte, Héraclès et les Dioscures interagissent dans le cadre du combat au Platanistas (c'est bien dans le temple des Dioscures au Phoibaion qu'avant ce combat les jeunes Spartiates sacrifient le chiot à Enyalios - HATZOPOULOS, 1971, p. 16-59) s'explique parce que - au milieu de l'histoire légendaire de la cité - Héraclès et les Dioscures remplissaient une fonction similaire, c'est-à-dire celle d'assurer (e. g. contre les visées des Hippocoontides ou des Apharetides) que le pouvoir royal du lignage de Tyndare restât solide ${ }^{34}$ - ce qui assure aux Dioscures aussi l'acquisition de leur statut d'immortels (Hom. Od. XI, 298-304 et Pind. Nem. X, 49-59).

C'est tout en tenant compte des exploits qui caractérisent aussi tout le cours de la vie d'Héraclès que peut, en effet, s'accomplir le destin de

33 Par exemple, Marguerite Yon (1992), de son côté, analyse les traces de la présence d'Héraclès à Chypre (ou, pour mieux le dire, d'un « Héraclès chypriote ») d'après les données dont on dispose, et observe entre autres la présence dans les documents iconographiques du motif du combat d'un homme contre un lion (patère d'Idalion ; Louvre AO 20134) - duquel l'épisode du lion de Némée qui voit comme protagoniste Héraclès est un exemple. Même - comme aussi Yon le relève de son côté - s'il n'est pas nécessaire d'identifier Héraclès dans ces images (p. 148), c'est bien l'inclusion du personnage d'Héraclès (par la fabrication du mythe du "lion de Némée ») dans le nombre des personnages qui " réalisent " ce motif fonctionnel du combat entre l'homme et le fauve (répandu dans plusieurs civilisations du bassin de la mer Méditerranée) qui nous intéresse. D'ailleurs (p. 149-54), Yon présente d'autres documents iconographiques proprement chypriotes (e. g. Met. Mus. 74.51.2455; Louvre AM 231) où l'identification du personnage représenté avec le type de l'Héraclès grec est presque sûre - grâce à ses attributs (notamment la peau de lion) - montrant en effet (p. 160) que l'Héraclès grec est connu à Chypre comme personnage hérö̈que confronté à des épreuves et des aventures. De son côté, Thierry Petit (2004) - à la suite de son analyse iconographique des reliefs du sarcophage d'Amathonte, qui représentent aussi des moments caractérisant la vie du dynaste (e. g. la chasse ; le combat ; les audiences ; le banquet ; le défilé des chars) - relève, par exemple, que les funérailles du dynaste jouaient un rôle essentiel du point de vue idéologique et que le défilé des chars renvoyait précisément au motif de l'apothéose d'Héraclès, pour indiquer l'héroïsation du dynaste défunt. Dans ce même système figuratif, est aussi remarquable la présence de quatre figures féminines du type de la déesse «Astarté »-en tant que divinité liée à la fertilité et à la protection de la vie et du roi/dieu -, qui sont aussi rapprochées par Petit dans son analyse à certains types figuratifs de déesses grecques que nous avons aussi mentionnées, telles qu' « Artémis » ou « Aphrodite ».

Ce serait aussi intéressant d'établir une comparaison entre la présence de certains éléments (e. g. le sphinx, le lion, la sirène) dans les reliefs chypriotes et leur présence dans les peintures des vases laconiens du $\mathrm{VI}^{\mathrm{e}}$ siècle av notre ère.

$34 \mathrm{Il}$ est intéressant de remarquer, à propos de cela, que les personnages représentés par des monuments au Platanistas (Paus. III, 15, 2-3) sont à peu près les mêmes que ceux mentionnés par Alcman dans l'une des premières sections d'un de ses parthénées (Alcm. fr. 3, 1-12 Calame). 
divinisation que la Pythie lui avait prophétisé au début de sa vie adulte ${ }^{35}-$ aussi implicite dans la descendance d'Héraclès de Zeus - et découvrir par conséquent (sous la perspective de la fabrication de la légende d'Héraclès ${ }^{36}$ ) aussi la synthèse entre le héros et, comme le relève P. Lévêque (LÉVÊQUE ; VERBANCK-PIÉRARD, 1992, p. 47 et 49-50), le palaios théos d'Hérodote dont il retrouve des homologues en Orient (II, 43-44).

\section{Les éléments du mythe grec de la naissance héracléenne des Scythes à la lumière des mythologies proche-orientales}

À la suite de ce que nous avons vu jusque-là, l'opportunité de rechercher des parallèles également parmi les protagonistes des légendes proche-orientales est une démarche désormais acquise par les chercheurs (e. g. BURKERT, 1992 ; HERMARY, 1992 ; BONNET, 1992). S'il est en effet évident désormais - d'après plusieurs études ${ }^{37}-$, par exemple, qu'Héraclès en tant que héros divinisé est étroitement lié au dieu phénicien Melqart, certaines affinités ont aussi été détectées avec certains sceaux mésopotamiens où un héros étrangle des serpents ou tue un monstre à plusieurs têtes (tel que l'Hydre), ou avec certains héros divinisés tels que Ninurta (le fils du dieu sumérien/akkadien Enlil - au rôle semblable à celui du grec Zeus, père d'Héraclès), lui aussi avec la peau de lion, la massue et l'arc, ou Gilgamesh (BURKERT, 1987, p. 119-27 et STAFFORD, 2012, p. 13).

D'une manière très synthétique, en abordant un champ d'études hors de notre spécialisation et dans les limites de cet article, on a choisi de mettre en évidence certains traits que deux héros proche-orientaux Aqht et Gilgamesh - semblent partager avec Héraclès, aussi par rapport à l'interaction avec certains dieux dans des circonstances analogues, à un moment donné de leur légende.

35 Voir aussi la prophétie de Tirésias dans Pind. Nem. I, 60-72.

36 Voir aussi LÉVÊQUE ; VERBANCK-PIÉRARD, 1992, p. 56-58.

37 Hors de toute prétention d'exhaustivité, je me limite à mentionner ici BONNET, 1988 et BONNET ; BRICAULT, 2016, p. 21-44, la section de JOURDAIN-ANNEQUIN, 1989, p. 95-170 consacrée à cette identification, et le chapitre de MALKIN, 2011 (2018), p. 171-200, dévolu à la même question. 


\section{A. Aqht}

Dans le poème homonyme d'Ugarit ${ }^{38}$, le jeune Aqht (l'héritier que $E l$ promet et Baal accorde enfin au héros Dan'el - un roi [?] ${ }^{39}$ - à la suite d'une période de sept jours, marquée par le sommeil qui se répète ${ }^{40}$ ) affronte la déesse Anat (Acte II, Scène 2, dans ce qu'on décrit comme la fête « pour son âge adulte ", MARGALIT, 1989, p. 123-26). Anat lui demande l'arc qu'il avait reçu de son père (dont la façon rappelle celle de l'arc scythique, et qu'Aqht se révèle capable de tendre) au moment d'être introduit à la chasse, en lui promettant enfin l'immortalité. Ayant accepté le sort des humains, Aqht se méfie de la déesse, qualifiée de vierge, en méprisant aussi ses ambitions de chasseresse (ce qui provoque la colère de la déesse). Aqht va donc mourir bientôt - ce qui comporte aussi la rupture de l'arc (Acte II, Scène 6) et le fait d'être dévoré par des oiseaux carnivores.

\section{B. Gilgamesh}

Dans le cas de Gilgamesh ${ }^{41}$ (héros semi-divin, roi porteur de culture $^{42}$ ) aussi - dont la légende (GEORGE, 2003, p. 3-70) est répandue en plusieurs versions (DALLEY, 2000, p. 45-47 et GEORGE, 2003, p. 159-375) dans différentes régions du Proche-Orient (e. g. BACHVAROVA, 2016, p. 54-77 et 78-110), en plus de la forme standard - à côté d'épisodes qui ont également du sens pour nous (e. g. les rites d'incubation, tablette IV, 11. 7-33; le combat contre le Taureau Céleste, tablette VI, 11. 113-50; le « chemin du soleil », tablette IX, 11. 136-96), nous relevons des moments comme celui de l'union entre Enkidu et Shamhat (tablette I, 11. 159-202), une

38 Je fais confiance essentiellement à l'édition réalisée par MARGALIT, 1989.

39 MARGALIT, 1989, p. 253 n'adhère pas à cette vue.

40 Ce qui représenterait un parallèle avec le sommeil d'Héraclès tel que nous l'avons décrit - tandis qu'on a aussi pensé à un rituel prolongé d'incubation.

41 Je fais confiance, pour le texte, essentiellement à DALLEY, 2000 et GEORGE, 2003.

42 Voir, en général, la première section de la tablette I : ça incluait la restauration des sanctuaires et des cultes, en particulier ceux d'Ishtar. 
prostituée sacrée de la déesse Ishtar, qui marque pour Enkidu le rejet de la vie sauvage dans la nature et le passage à la civilisation et à la culture ; ce qui associé à sa connaissance du monde sauvage - lui permet aussi de protéger le protagoniste (tablette III, 11. 6-12, 131-35, 218-27 ; V, 11. 85-94, 156-58 et 181-91). Par ailleurs, le moment de sa mort marquera, pour lui, la conclusion d'une vie digne de mémoire (tablette VIII), comme d'ailleurs ce sera le cas pour Gilgamesh à la suite de sa quête d'immortalité et de son acquisition de sagesse (tablette $\mathrm{XI}^{43}$; d'une manière semblable à Héraclès - e. g. Diod. IV, 38,4 - lorsque c'est bien grâce à la mort du héros qu'il gagne définitivement son statut divin) ${ }^{44}$. Un épisode aussi remarquable (par rapport à ce dernier détail) est celui de la rencontre de Gilgamesh avec Ishtar ${ }^{45}$ et le refus qu'il oppose à la proposition de la déesse de devenir son épouse (tablette VI, 11. 6-79) : un reproche à propos du destin de tous les amants de la déesse qui contient aussi certaines analogies avec le refus d'Aqht de donner son arc à Anat (essentiellement, la méfiance du héros envers toute promesse de rachat de sa condition humaine, si on considère e. g. le cas de Tammuz, et qui rappelle aussi certains avis propres à la sagesse grecque, e. g. Alcm. fr. 3, 16-21, Calame).

Ce dernier cas - si on tient compte de ce qu'Enkidu était à l'origine et de l'interaction qu'il y aura ensuite entre lui et Gilgamesh - est aussi remarquable, je crois, pour vérifier (en nous rapprochant des conclusions) un aspect nodal que nous avons essayé de mettre en évidence dans cette étude, c'est-à-dire la relation qui court entre un système d'exigences (et de fonctions) partagées par plusieurs civilisations (qui, dans notre cas, sont en contact dans le contexte environnemental de la mer Méditerranée) et la façon différente selon laquelle elles se configurent dans les formes de société de chaque civilisation (aussi bien que dans l' " ordre du récit» des mythes que chaque civilisation fabrique - au sein duquel ces mêmes fonctions sont organisées de manière différente entre certains types de héros qui doivent les remplir).

43 Le détail du sommeil est intéressant par rapport à cette nature mortelle, 11. 205-41.

44 C'est bien à ce propos que nous pouvons rappeler cette idée-là d'un « ordre du récit », dans le cadre duquel une série d'éléments culturels (fonctions/notions) analogues est néanmoins organisée de manière différente et entre personnages et moments différents.

45 Quelques hypothèses au sujet de certaines analogies entre Ishtar/Inanna et certains aspects de la déesse des Grecs Aphrodite sont avancées, en général, dans PENGLASE, 1994, p.1-12 et 134-51, et METCALF, 2015, p. 171-90. 
Par exemple - pour ce qui concerne le rapport entre la dimension humaine (e. g. mortelle, civilisée) et celle hors de l'humain (e. g. exempte de la mort, sauvage) -, la relation qui lie Enkidu et Gilgamesh (autour de laquelle se construit une partie importante du récit) nous révèle des dynamiques semblables à celles qui se déroulent au cours de la légende d'Héraclès (par rapport à la fonction qu'il doit remplir dans chaque épisode de cette même légende), mais qui se configurent bien dans un "ordre du récit » et sous une forme différents. Cela, je pense, s'avère d'autant plus évident aussi à la lumière des documents matériels (e. g. des objets, comme l'arc, ou des types figuratifs analogues, comme celui de l'homme à la massue et à la peau de lion) qui reviennent dans ces civilisations en renvoyant à certains aspects de la société (e. g. le rôle de la chasse), et qui montrent dans leur diffusion, précisément, le réseau de contacts entre ces différentes civilisations .

S'il semblerait donc (lorsqu'on tient compte e. g. de l'attribut de la massue qui l'identifie) qu'au long de la série de ses exploits Héraclès lui-même n'ait pas été totalement étranger à la dimension sauvage avec laquelle il entre en contact (ce qui lui est nécessaire pour qu'il puisse réussir ses tâches) -, dans le cas d'Enkidu et Gilgamesh c'est grâce à Gilgamesh qu'Enkidu est intégré dans la vie civilisée (à la suite de l'union avec Shamhat - ce qu'implique aussi qu'il devient mortel) mais, d'un autre côté, c'est bien par le fait qu'Enkidu avait connu la dimension sauvage qu'il devient déterminant pour l'achèvement des exploits de Gilgamesh (e. g. le combat contre Humbaba) - ce qui conduit enfin Gilgamesh à l'acquisition de son propre statut de héros.

\section{Conclusion. Un héros «de la mer corruptrice» : logiques méditerranéennes dans l'identité héroïque d'Héraclès}

À la suite de ce que nous avons vu jusque-là, nous pourrions ainsi choisir une définition d'Héraclès comme celle d'un héros « de la mer corruptrice ", tout en intégrant dans ce choix le sens du titre d'une étude désormais très célèbre du début des années 2000 (HORDEN ; PURCELL), qui soulevait la question de la mer Méditerranée comme d'un bassin qui a permis d'établir, à plusieurs niveaux (notamment si on y inclut aussi la mer Noire), 
des réseaux de connexions et d'échanges multiples (e. g. DOMMELEN ; KNAPP (éds.), 2010 et KNAPP ; DEMESTICHA, 2017, notamment, pour les buts de cet article, l'Introduction et les Conclusions) entre civilisations différentes (qu'on dirait être aussi à l'origine du véritable « tissu » historique de chacune de ces mêmes civilisations), en permettant aux mythes eux-mêmes de voyager (AUDLEY-MILLER ; DIGNAS (dir.), 2018), et en ouvrant aussi une nouvelle série d'enquêtes qui a enrichi notre perception de la complexité d'une définition organique de cette action $《$ corrompante $»^{46}$.

On peut donc conclure que certains cas - comme celui du mythe de la naissance du peuple des Scythes tel que retenu par les Grecs de la mer Noire et transmis par Hérodote - deviennent, pour nous, des objets précieux d'analyse dans le but de saisir au moins l'existence de ce « code culturel» commun que nous avons évoqué.

Comme nous avons eu la possibilité de l'observer - au fur et à mesure qu'Héraclès achève ses exploits -, il construit aussi son identité (ou, si on veut, le système de fonctions qui l'identifie) de héros pour les Grecs, de façon cohérente avec ce que la Pythie lui avait prophétisé. Mais - au niveau de la « mise en ordre » des récits qui le concernent -, cela comporte aussi que sa légende soit façonnée sous certaines formes données. Ainsi, on a vu d'ailleurs que certains éléments qui interviennent dans sa légende (e. g. créatures monstrueuses, êtres surhumains, régions à l'altérité marquée ; mais aussi agents divins en concurrence) - et qui deviennent essentiels pour la définition du personnage d'Héraclès en tant que héros - reviennent aussi dans des légendes centrées sur d'autres personnages qui sont des expressions du monde grec (e. g. Ulysse, Phrixos, Jason) ou bien d'autres civilisations dans le bassin de la mer Méditerranée (nous avons choisi les cas d'Aqht et de Gilgamesh).

En outre - par rapport à d'autres héros grecs que nous venons de mentionner -, il faut aussi considérer que l'ampleur du champ d'action d'Héraclès, dans des régions où il exerce une fonction active par rapport à la situation préexistante, rend sa légende d'autant plus digne de mention à une échelle méditerranéenne, au point qu'au niveau de la figuration à l'époque archaïque il est pleinement intégré dans des types figuratifs (e. g. l'homme à la peau de lion - notamment HERMARY, 1992 et YON, 1992,

46 Je me limite ici à rappeler HARRIS (dir.), 2005 ; MALKIN (dir.), 2005 ; MALKIN, 2011 (2018) et, pour les aspects des pratiques religieuses en particulier, CHANIOTIS (éd.), 2011. 
à bien considérer à la lumière du témoignage d'Hérodote [Hdt. II, 43-44]) amplement répandus et, ensuite (par exemple, à l'époque hellénistique), il est lui-même « exporté » et réinterprété à l'extérieur du monde grec (notamment BONNET, 1992 et WOOD, 2018). Il est vrai que - puisque le sujet de cet article a été le récit hérodotéen de la naissance des Scythes - nous avons eu tendance à concentrer notre attention presque exclusivement sur la partie orientale du bassin méditerranéen (mer Égée et, notamment, mer Noire et Proche-Orient). Il faut cependant ne pas oublier que les exploits d'Héraclès ont également été remarquables dans les régions occidentales de la mer Méditerranée ${ }^{47}$.

Il ne s'agit pas non plus, pour autant (comme nous l'avions proposé en début d'analyse), seulement de considérer Héraclès comme l'outil par lequel les Grecs portaient leur regard sur certains régions ou peuples qui, auparavant, leur étaient inconnus mais aussi (en retournant notre propre regard) comme l'un des produits les plus riches que le monde grec ait apportés à la formation d'un système de valeurs culturelles partagées à l'intérieur du bassin de la Méditerranée.

\section{Bibliographie.}

ALY, Wolf. Volksmärchen, Sage und Novelle bei Herodot und seinen Zeitgenossen, Göttingen 1969 (2 édition).

AUDLEY-MILLER, Lucy ; DIGNAS, Beate (dir.). Wandering Myths: Transcultural Uses of Myth in the Ancient World. Berlin - Boston: Walter de Gruyter, 2018.

BACHVAROVA, Mary R. From Hittite to Homer: The Anatolian Background of Ancient Greek Epic. Cambridge : Cambridge University Press, 2016.

BADER, Françoise. Les Travaux d'Héraclès et l'idéologie tripartie. In : BONNET, Corinne - JOURDAIN-ANNEQUIN, Colette (éds.) 1992, p. 7-42. 
BADER, Françoise. Héraclès et le cheval. In : BONNET, Corinne ; JOURDAIN-ANNEQUIN, Colette ; PIRENNE-DELFORGE, Vinciane (éds.). p. 135-53.

BONNET, Corinne. Melqart : Cultes et mythes de l'Héraclès tyrien en Méditerranée. (Studia Phœnicia 8 ; Bibliothèque de la faculté de philosophie et lettres de Namur 69). Louvain-Namur : Peeters, 1988.

BONNET, Corinne. Héraclès en Orient : interprétations et syncrétismes. In : BONNET, Corinne ; JOURDAIN-ANNEQUIN, Colette (éds.). 1992, p. 165-98.

BONNET, Corinne; BRICAULT, Laurent. Quand les dieux voyagent : cultes et mythes en mouvement dans l'espace méditerranéen antique. Genève : Labor et Fides, 2016.

BONNET, Corinne ; JOURDAIN-ANNEQUIN, Colette ; PIRENNEDELFORGE, Vinciane (éds.). Le Bestiaire d'Héraclès : III ${ }^{e}$ Rencontre héracléenne. (Kernos, supplément 7). Liège: Presses universitaires de Liège, 1998.

BONNET, Corinne ; JOURDAIN-ANNEQUIN, Colette (éds.). Héraclès : d'une rive à l'autre de la Méditerranée. Bilan et perspectives, Actes de la table ronde de Rome, 15-16 septembre 1989 à l'occasion du cinquantenaire de l'Academia Belgica, en hommage à Franz Cumont, son premier président. Rome : Institut Historique Belge de Rome, 1992.

BRILlANTE, Carlo. La paideia di Eracle. In: BONNET, Corinne JOURDAIN-ANNEQUIN, Colette (éds.) 1992, p. 199-222.

BURKERT, Walter. Oriental and Greek Mythology: The Meeting of Parallels. In : Interpretations of Greek Mythology. Edited by Jan BREMMER. London: Routledge, 1987, p. 10-40.

BURKERT, Walter. Eracle e gli altri eroi culturali del Vicino Oriente. In: BONNET, Corinne - JOURDAIN-ANNEQUIN, Colette (éds.). 1992, p. 111-27.

BURKERT, Walter. Héraclès et les animaux. Perspectives préhistoriques et pressions historiques. In : BONNET, Corinne ; JOURDAIN-ANNEQUIN, Colette ; PIRENNE-DELFORGE, Vinciane (éds.). 1998, p. 8-21

CASSIRER, Ernst. Philosophie der symbolischen Formen. Première partie : Die Sprache. Berlin : Bruno Cassirer, 1923. 
CHANIOTIS, Angelos (éd.). Ritual Dynamics in the Ancient Mediterranean. Agency, Emotion, Gender, Representation. Stuttgart: Franz Steiner Verlag, 2011.

CORCELLA, Aldo. Commento. In: Erodoto, Le Storie: Volume IV, Libro IV, La Scizia e la Libia. Introduzione e commento di Aldo Corcella. Testo critico di Silvio M. Medaglia. Traduzione di Augusto Fraschetti. Milano: Mondadori (Fondazione Lorenzo Valla), 1993.

DALLEY, Stephanie. Myths from Mesopotamia Creation, the Flood, Gilgamesh, and Others. Revised edition. Oxford: Oxford University Press, 2000.

DEVEREUX, Georges. The Kolaxaian horse of Alkman's Partheneion. In: CQ 15 (1965), p. 176-184.

DOMMELEN, Peter van ; KNAPP, A. Bernard (éds.). Material Connections in the Ancient Mediterranean: Mobility, Materiality and Mediterranean Identities. Oxon - New York : Routledge, 2010.

ELLINGER, Pierre. Artémis, déesse de tous les dangers. En collaboration avec M. Dennehy. Paris : Larousse, 2009.

ELLIS-EVANS, Aneurin. The Kingdom of Priam: Lesbos and the Troad between Anatolia and the Aegean. Oxford: Oxford University Press, 2019.

FINKELBERG, Margalit. Greeks and Pre-Greeks: Aegean Prehistory and Greek Heroic Tradition. Cambridge: Cambridge University Press, 2005.

GEORGE, A. R. The Babylonian Gilgamesh Epic: Introduction, Critical Edition And Cuneiform Texts. Volume I. Oxford : Oxford University Press, 2003.

GERNET, Louis ; BOULANGER, André. Le génie grec dans la religion, Paris : Albin Michel, 1932 (1970), p. 221-231.

GERNET, Louis. Mariages de tyrans. In : Hommage à Lucien Febvre, Paris : 1954, p. 41-53 ; ensuite in : IDEM, Anthropologie de la Grèce antique. Paris : Maspero, 1968, p. 344-59.

GIANGIULIO, Maurizio. Greci e non Greci in Sicilia alla luce dei culti e delle leggende di Eracle. In : Modes de contacts et processus de transformation dans les sociétés anciennes. Actes du colloque de Cortone (24-30 mai 1981). Rome : Ecole française de Rome, 1983, p. 785-846. 
GNOLI, Gerardo ; VERNANT, Jean-Pierre (éds.). La Mort, les morts dans les sociétés anciennes. Paris: Éd. de la Maison des sciences de l'Homme, 1982 ; Cambridge : Cambridge University Press, 1982 (1990).

HARRIS, William V. (dir.). Rethinking the Mediterranean. Oxford: Oxford University Press, 2005.

HARTOG, François. Le miroir d'Hérodote. Essai sur la représentation de l'autre. Paris : Gallimard, 1981 (2001).

HARTOG, François. La mort de l'autre : les funérailles des rois scythes. In : GNOLI, Gerardo ; VERNANT, Jean-Pierre (éds.). 1982 (1990), p. 143-54.

HATZOPOULOS, Miltiades. Le Culte des Dioscures et la double Royauté à Sparte, Thèse dactylographiée de $3^{\mathrm{e}}$ Cycle (EPHE, Vème section - Sciences Religieuses) préparée sous la direction de M. Jean-Pierre Vernant, soutenue le 13 mai 1971. Paris : 1971.

HERMARY, Antoine. Quelques remarques sur les origines proche-orientales de l'iconographie d'Héraclès. In : BONNET, Corinne - JOURDAINANNEQUIN, Colette (éds.). 1992, p. 129-43.

HINGE, George. Scythian and Spartan Analogies in Herodotus' Representation: Rites of Initiation and Kinship Groups. In: The Cauldron of Ariantas. Studies presented to A.N. Ščeglov on the occasion of his 70th birthday. Edited by Pia Guldager Bilde, Jakob Munk Højte and Vladimir F. Stolba. Aarhus 2003, p. 55-74.

HODKINSON, Stephen (éd.). Sparta: Comparative approaches. Swansea: Classical Press of Wales, 2009.

HORDEN, Peregrine ; PURCELL, Nicholas. The Corrupting Sea: A Study of Mediterranean History, Oxford: Blackwell, 2000.

JOURDAIN-ANNEQUIN, Colette. Héraclès aux portes du soir : Mythe et histoire. Annales Littéraires de l'Université de Besançon, $\mathrm{n}^{\circ}$ 402. Paris : Diffusion Les Belles Lettres, 1989.

KNAPP, Bernard ; DEMESTICHA, Stella. Mediterranean Connections: Maritime Transport Containers and Seaborne Trade in the Bronze and Early Iron Ages. With contributions by Robert Martin and Catherine E. Pratt. New York - Oxon: Routledge, 2017. 
LE GOFF, Jacques ; VERNANT, Jean-Pierre. Dialogues sur l'histoire : Entretiens avec Emmanuel Laurentin. Montrouge : Bayard Éditions, 2014. LÉVÊQUE, Pierre ; VERBANCK-PIÉRARD, Annie. Héraclès héros ou dieu ?. In : BONNET, Corinne ; JOURDAIN-ANNEQUIN, Colette (éds.) 1992, p. 43-65.

MAGNELLI, Adalberto. Commento storico al Libro IV. In: MARIOTTA, Giuseppe ; MAGNELLI, Adalberto, Diodoro Siculo. Biblioteca storica: Libro IV: Commento storico. Milano: Vita e Pensiero, 2012.

MALKIN, Irad. A small Greek world: Networks in the ancient Mediterranean. Oxford: Oxford University Press, 2011 (trad. fr. Un tout petit monde: Les réseaux grecs de l'Antiquité. Paris: Les Belles Lettres, 2018)

MALKIN, Irad (dir.). Mediterranean Paradigms and Classical Antiquity (Special issue of Mediterranean Historical Review). London: Routledge, 2005.

MARGALIT, Baruch. The Ugaritic Poem of Aqhat (Beihefle zur Zeilschrift für die alttestamentiliche Wissenschaft, 182). Berlin - New York: Walter de Gruyter, 1989.

MARIOTTA, Giuseppe. Commento storico al Libro IV. In: MARIOTTA, Giuseppe ; MAGNELLI, Adalberto, Diodoro Siculo. Biblioteca storica: Libro IV: Commento storico. Milano: Vita e Pensiero, 2012.

MAUSS, Marcel. Une catégorie de l'esprit humain : la notion de personne et celle de «moi». In : Journal of the Royal Anthropological Institute LXVIII (1938), Londres (Royal Memorial Lecture, 1938), ensuite dans Mauss 1950, p. 331-62.

MAUSS, Marcel. Sociologie et anthropologie. Paris : Presses Universitaires de France, 1950.

MAYOR, Adrienne. The Amazons : lives and legends of warrior women across the ancient world. Princeton - Oxford : Princeton University Press, 2014.

METCALF, Christopher. The Gods Rich in Praise: Early Greek and Mesopotamian Religious Poetry. Oxford: Oxford University Press, 2015.

MILLENDER, Ellen. Herodotus and Spartan despotism. In: POWELL, Anton ; HODKINSON, Stephen (éds.). 2002, p. 1-61.

MILLENDER, Ellen. The Spartan Dyarchy: a comparative perspective. In: HODKINSON, Stephen (éd.). 2009, p. 1-67 
NILSSON, Martin P. The Mycenaean Origin of Greek Mythology. Berkeley: University of California Press, 1932.

PAPADOPOULOU, Thalia. Heracles and Euripidean Tragedy. Cambridge: Cambridge University Press, 2005.

PARKER, Robert (éd.). Changing Names: Tradition and Innovation in Ancient Greek Onomastics. Proceedings of the British Academy 222. Oxford: Oxford University Press, for the British Academy, 2019.

PENGLASE, Charles. Greek Myths and Mesopotamia: Parallels and Influence in the Homeric Hymns and Hesiod. New York: Routledge, 1994.

PETIT, Thierry. Images de la royauté amathousienne: le sarcophage d'Amathonte. In : PERRIN, Y. ; PETIT, Th. (éd.), Iconographie impériale, iconographie royale, iconographie des élites dans le monde gréco-romain. Publications de l'Université de Saint-Etienne, 2004, p. 49-96.

PIRENNE-DELFORGE, Vinciane. Des épiclèses exclusives dans la Grèce polythéiste ? L'exemple d'Ourania. In : Nommer les Dieux : théonymes, épithètes, épiclèses dans l'Antiquité. Textes réunis et édités par Nicole BELAYCHE [et al.]. Turnhout : Brepols - Rennes : Presses universitaires de Rennes, 2005, p. 271-90.

PIRENNE-DELFORGE, Vinciane ; PIRONTI, Gabriella, L'Héra de Zeus: Ennemie intime, épouse définitive. Paris: Les Belles Lettres, 2016.

PIRONTI, Gabriella. Au nom d'Aphrodite: Réflexions sur la figure et le nom de la déesse née de l'aphros. In : Nommer les Dieux : théonymes, épithètes, épiclèses dans l'Antiquité. Textes réunis et édités par Nicole BELAYCHE [et al.]. Turnhout : Brepols - Rennes : Presses universitaires de Rennes, 2005, p. $129-42$.

PIRONTI, Gabriella. Entre ciel et guerre: figures d'Aphrodite en Grèce ancienne. Liège: Centre International d'Étude de la Religion Grecque Antique, 2007.

POWELL, Anton ; HODKINSON, Stephen (éds.). Sparta: beyond the mirage. Swansea: Classical Press of Wales, 2002.

SCHNAPP-GOURBEILLON, Annie. Les lions d'Héraklès. In: BONNET, Corinne - JOURDAIN-ANNEQUIN, Colette ; PIRENNE-DELFORGE, Vinciane (éds) 1998, p. 98-112. 
STAFFORD, Emma. Herakles. London - New York: Routledge, 2012.

TALAMO, Clara. La Lidia Arcaica: Tradizioni genealogiche ed evoluzione istituzionale. Bologna: Patron, 1979.

VERNANT, Jean-Pierre. Aspects de la personne dans la religion grecque ». Exposé fait au colloque " Problèmes de la personne » organisé par le Centre de recherches de psychologie comparative, Royaumont 29 septembre -3 octobre 1960. Ensuite in : Vernant, 1965 (1985), p. 355-70.

VERNANT, Jean-Pierre. Mythe et pensée chez les Grecs : Études de psychologie historique. Paris : Maspero, 1965 (nouvelle édition augmentée Paris : La Découverte, 1985).

WATHELET, Paul, Héraklès. le monstre de Poséidon et les chevaux de Tros. In : BONNET, Corinne ; JOURDAIN-ANNEQUIN, Colette ; PIRENNEDELFORGE, Vinciane (éds.). 1998, p. 52-64.

WOOD, Rachel. Wandering Hero, Wandering Myths? The Image of Heracles in Iran. In: AUDLEY-MILLER, Lucy - DIGNAS, Beate (dir.). 2018, p. 327-55.

YON, Marguerite. Héraclès à Chypre. In: BONNET, Corinne ; JOURDAINANNEQUIN, Colette (éds.) 1992, p. 145-60.

RECEBIDO EM: 05/09/2020

APROVADO EM: 01/10/2020 